\title{
Peccei-Quinn symmetry breaking via asymptotically safe dynamical scalegenesis: a walking axicolor and axion
}

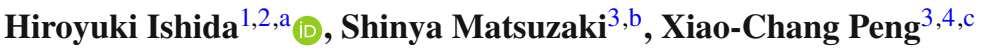 \\ ${ }^{1}$ Theory Center, IPNS, KEK, Tsukuba, Ibaraki 305-0801, Japan \\ ${ }^{2}$ Center for Liberal Arts and Sciences, Toyama Prefectural University, Toyama 939-0398, Japan \\ ${ }^{3}$ Center for Theoretical Physics and College of Physics, Jilin University, Changchun 130012, China \\ ${ }^{4}$ Department of Physics, Brown University, Providence, RI 02912, USA
}

Received: 25 November 2021 / Accepted: 10 January 2022 / Published online: 3 February 2022

(C) The Author(s) 2022

\begin{abstract}
Breaking the Pecci-Quinn (PQ) symmetry by the perturbative dynamics would suffer from a hierarchy problem, just like the electroweak symmetry breaking in the standard model. The dynamics of the axion, associated with the PQ symmetry breaking, would also involve a triviality problem. We provide a paradigm to resolve those two problems potentially existing in the PQ symmetry breaking scenario, with keeping the successful axion relaxation for the QCD strong CP phase. The proposed theory includes an axicolor dynamics with the axicolored fermions partially gauged by the QCD color, and is shown to be governed by an asymptotically safe (AS) fixed point: quantum scale invariance is built. The AS axicolor is actually a "walking" dynamics, which dynamically breaks a PQ symmetry, a part of the chiral symmetry carried by the axicolored fermions. The PQ scale generation is then triggered by the nonperturbative dimensional transmutation in the "walking" dynamics. A composite axion emerges as the associated Nambu-Goldstone boson. That is, no hierarchy or triviality problem is present there. The composite axion can potentially be light due to the characteristic feature of the AS axicolor ("walking" axicolor), becomes the QCD axion in the anti-Veneziano limit, and gets heavier by the subleading correction. The composite axion relaxes the QCD theta parameter, involving heavier relaxation partners such as axicolored pseudoscalar mesons, and the ultraviolet correction to the relaxation mechanism is protected by the established (near) scale invariance during the "walking" regime.
\end{abstract}

\footnotetext{
a e-mail: hiroyuki403@gmail.com (corresponding author)

b e-mail: synya@jlu.edu.cn

c e-mail: xiaochang_peng@brown.edu
}

\section{Introduction}

Violation of CP symmetry was theoretically predicted and observed in the weak interactions, however, not observed in the strong interactions, which is called strong CP problem. (See e.g., [1-3] as reviews.) One of the most attractive solution to this problem is introducing a global symmetry, which is often called Peccei-Quinn (PQ) symmetry [1,4-6] $U(1)_{\mathrm{PQ}}$, and the pseudo Nambu-Goldstone boson associated with the spontaneous breaking of this global symmetry is called axion.

The role of the spontaneous breaking of the PQ symmetry can simply be played by a new Higgs field (a PQ-breaking scalar), via an assumed double-well type potential, analogously to the electroweak symmetry breaking via the Higgs field of the standard model [2,7-9]. However, this kind of simple and perturbative scenario inevitably suffers from a hierarchy problem or instability for the PQ breaking scale $\left(f_{a}\right)$ against power corrections arising from the Planck scale, similarly to the gauge hierarchy problem caused by the Higgs boson in the standard model. ${ }^{1}$

Furthermore, renormalization group evolution for the PQbreaking scalar sector would exhibit a Landau pole feature at a finite ultraviolet scale because of the scalar quartic coupling. This implies that the PQ scalar theory as a continuum quantum field theory is nothing but non-interacting and trivial, which is characterized by the Gaussian-infrared fixed point with the vanishing scalar quartic coupling, at only which the theory is renormalizable. This problem, called triviality, generically involves scalar theories in four-dimension, and QED-like $U(1)$ gauge theories, as present in the standard

\footnotetext{
${ }^{1}$ Moreover, quantum gravity would be expected to break any global symmetry, which could destabilize the axion relaxation mechanism [1013]. Later we will give comments on this issue in terms of the presently proposed framework.
} 
model. Namely, the perturbative PQ breaking causes another triviality problem, which needs to be improved or replaced by more fundamental theories, otherwise the theory giving the PQ breaking is not realized in a pregnant framework of the continuum theory. This problem would still be left even if the PQ symmetry is realized as an accidental unbroken symmetry arising from a gauge symmetry breaking [14-29], where the new gauge coupling may also have a Landau pole.

A simple solution to two problems is to explain the origin of PQ symmetry breaking by a strong dynamics, called axicolor [30,31]. In this case, the PQ symmetry is realized as a part of the global chiral symmetry carried by the axicolored fermions, and is spontaneously broken by the dynamical generation of the fermion mass and nonzero chiral condensate due to the strongly coupled axicolor dynamics, just like QCD. Thus, an axion arises as a composite Nambu-Goldstone boson associated with the spontaneous chiral breaking, and therefore, no hierarchy, or triviality problem for the PQ-breaking sector is present there. ${ }^{2}$

As for the triviality problem, the author in Ref. [34] has recently found perturbatively asymptotic-free fixed points for all the couplings in an axion model. However, in such a perturbative approach the hierarchy problem for the PQbreaking scale has been unsolved.

In this paper, we propose a new possibility that realizes a composite axion in asymptotically non-free, but nontrivially interacting ultraviolet gauge theory, characterized by an asymptotically safe (AS) fixed point manifestly keeping the quantum scale invariance. This provides a new paradigm to resolve the hierarchy and triviality problems for the axion dynamics, without spoiling the successful axion relaxation for the QCD strong CP problem.

The theory includes the gauge (called axicolor), Yukawa and scalar sectors. We work on the $\left(1 / N_{F}, 1 / N_{C}\right)$ dual expansion with $N_{F} / N_{C}$ fixed and $N_{F} \gg N_{C}$ (which was dubbed anti-Veneziano limit in the literature [35]), where $N_{F}$ and $N_{C}$ respectively denote the numbers of flavors and colors. We then see that the axion arises as a composite pseudo-NambuGoldstone boson associated with the PQ symmetry breaking, a part of the chiral symmetry breaking for $N_{F}$ fermions in the strong-gauge coupling regime of the axicolor. The spontaneous breaking of the chiral symmetry is governed by a strong QED like dynamics with the constant coupling identified as the AS fixed point coupling, that is almost scale-invariant, walking dynamics. That is, no hierarchy or triviality problem is present in the PQ breaking scenario.

The composite axion becomes the QCD axion in the antiVeneziano limit, and can be heavier due to the subleading nature. The phenomenologically viable relaxation mecha-

\footnotetext{
${ }^{2}$ For similar frameworks breaking the PQ symmetry, realized as a part of composite chiral symmetry, by the dimensional transmutation, e.g. see $[29,32,33]$.
}

nism for the strong $\mathrm{CP}$ phase works at the subleading order of the large $N_{F}$ limit. The QCD CP problem can thus be interpreted as the subleading phenomenon, and solved by a hybrid relaxation mechanism involving a couple of (composite) pseudoscalars, like so-called the ultraviolet solution [36-39].

Several comments and discussions along the presently proposed scenario are provided in Sect. 6.

\section{Asymptotically safe axicolor}

The axicolor is introduced as the gauge symmetry of an $S U\left(N_{C}\right)$ group with the gauge coupling $g$, to which $N_{F^{-}}$ fundamental representation Dirac fermions couple. We vectorlikely gauge the chiral symmetry in part by the QCD charge, and identify the QCD color triplet as three of $N_{F}$ Dirac fermions, such that the $N_{F}$-dimensional vector formed by the total $N_{F}$ Dirac fermions goes like

$F=\left(\psi^{\alpha}, \psi_{1}, \ldots, \psi_{n_{F}}\right)^{T}$

with $\alpha=1,2,3$ (QCD colors) and $n_{F}=N_{F}-3$. This way of charging the QCD colors is a generalization of the dynamical axion model in [31], which corresponds to $n_{F}=1$.

We also introduce QCD color-singlet scalar fields which take an $n_{F} \times n_{F}$ matrix form $(M)$ transforming in a bifundamental way under $U\left(n_{F}\right)_{L} \times U\left(n_{F}\right)_{R}$. This chiral scalar matrix $M$ can only have the quartic potential terms because of the scale symmetry: ${ }^{3}$

$V_{M}=\lambda_{1} \operatorname{tr}\left[\left(M^{\dagger} M\right)^{2}\right]+\lambda_{2}\left(\operatorname{tr}\left[M^{\dagger} M\right]\right)^{2}$,

with the quartic couplings $\lambda_{1}$ and $\lambda_{2}$.

The axicolored fermions do not allow to have Dirac masses because of the scale invariance. Instead, they couple to $M$ in an invariant way under the scale symmetry and the chiral $U\left(n_{F}\right)_{L} \times U\left(n_{F}\right)_{R}$ symmetry:

$\mathcal{L}_{y}=-y\left(\bar{\psi}_{L} M \psi_{R}+\bar{\psi}_{R} M^{\dagger} \psi_{L}\right)$,

where $\psi=\left(\psi_{1}, \ldots, \psi_{n_{F}}\right)^{T}$ corresponding to the subset of $F$ in Eq. (1). The Yukawa coupling $y$ can always be taken to be real, by field redefinition of $\psi_{L, R}$.

For later convenience, we define the fine structure constants relevant to the anti-Veneziano limit:

$$
\alpha_{g} \equiv \frac{N_{C} g^{2}}{(4 \pi)^{2}}, \quad \alpha_{y} \equiv \frac{N_{C} y^{2}}{(4 \pi)^{2}},
$$

\footnotetext{
3 The null mass term as well as other vanishing dimensionful coupling terms at the the Planck scale can be ensured by possible existence of another AS fixed point over the Planckian scale and its associated large anomalous dimensions, which enables to tune those to be zero [40-44].
} 
$\alpha_{\lambda_{1}} \equiv \frac{n_{F} \lambda_{1}}{(4 \pi)^{2}}, \quad \alpha_{\lambda_{2}} \equiv \frac{n_{F}^{2} \lambda_{2}}{(4 \pi)^{2}}$

All of them are of $O\left(N_{F}^{0}, N_{C}^{0}\right)$ in the large $N_{F}$ and $N_{C}$ limit.

The Yukawa coupling $y$ is assumed to be tiny in the AS domain (e.g., $10^{-90} \lesssim \alpha_{y} \lesssim 10^{-6}$ for a later reference) and gets asymptotically free. The QCD coupling can reach the asymptotically-free fixed point, because the gluonic contribution still dominates over colored fermion's even including extra $\psi^{\alpha}$ in Eq. (1). Since the Yukawa coupling is tiny, the scalar sector will be almost completely external to the axicolor dynamics, so will also become irrelevant to the dynamics of emergent composite axion itself.

Of particular interest is in the strongly coupled regime for the axicolor, which would give rise to the spontaneous breaking of the chiral $U\left(N_{F}\right)_{L} \times U\left(N_{F}\right)_{R}$ symmetry. Then the axicolor dynamics responsible for the chiral symmetry breaking can be viewed like a strong QED with the constant gauge coupling. In that case, for the dynamical-chiral symmetry breaking, we may adopt the result based on the ladder Schwinder-Dyson gap equation, which has extensively been studied in several contexts [45-53] (see also a review [54].). In terms of the current coupling convention in Eq. (4), the critical coupling and the generated fermion dynamical mass $\left(m_{F}\right)$, following so-called the Miransky scaling [50] (similar to the Berezinsky-Kosterlitz-Thouless scaling), read

$$
\begin{aligned}
& \alpha_{g}^{\mathrm{cr}}=\frac{N_{C}}{4 \pi} \cdot \frac{\pi}{3} \cdot\left(\frac{2 N_{C}}{N_{C}^{2}-1}\right)=\frac{1}{6} \cdot\left(\frac{N_{C}^{2}}{N_{C}^{2}-1}\right) \simeq \frac{1}{6}, \\
& m_{F} \simeq 4 M_{\mathrm{pl}} \exp \left[-\frac{\pi}{\sqrt{\frac{\alpha_{g}}{\alpha_{g}^{\mathrm{cr}}}}-1}\right],
\end{aligned}
$$

where the ultraviolet scale has been set to the Planck scale $M_{\mathrm{pl}}$.

As one reference, we may identify the size of the critical coupling as an AS fixed point $\alpha_{g}^{*}$, which has been predicted in the large $N_{F}$ gauge theory [55]: ${ }^{4} \alpha_{g}^{*}=3 N_{C} /\left(2 N_{F}\right)$. In that case we would have

$N_{F}=9 N_{C}\left(1-\frac{1}{N_{C}^{2}}\right) \simeq 9 N_{C}$.

\footnotetext{
$\overline{4}$ To be more precise, the validity of the large $N_{F}$ fixed point has been thoroughly questioned [56], where it is explained why the fixed point is an artefact of the infinite $N_{F}$ limit, and incompatible with any finite set of higher-order corrections at finite $N_{F}$. This conclusion is also corroborated by advanced lattice simulations [57], where no indications for an ultraviolet fixed point of the above form have been found. At any rate, our main claim on the emergence of composite axion and the successful relaxation mechanism will not substantially be affected even if the gauge fixed point is unwarranted in the perturbative coupling regime.
}

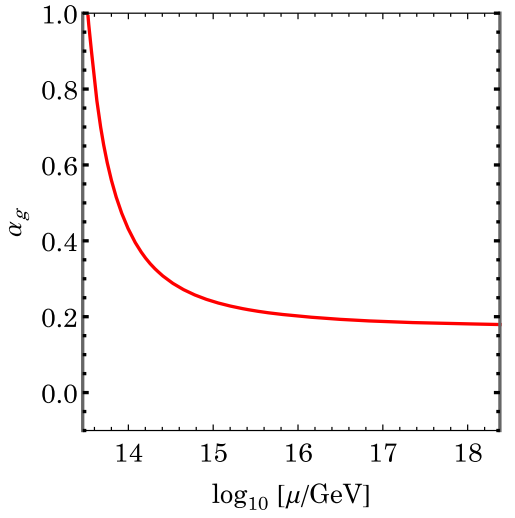

Fig. 1 Renormalization evolution of the axicolor coupling $\alpha_{g}$ from the fermion dynamical mass scale $m_{F}$ up to the Planck scale $M_{\mathrm{pl}} \simeq$ $2.4 \times 10^{18} \mathrm{GeV}$ in the strong coupling regime, in the anti-Veneziano limit $\left(N_{F}, N_{C} \gg 1\right.$ with $\left.N_{F} / N_{C} \gg 1\right)$. As a reference, the numbers of axicolors $N_{C}$ and axicolored fermions $N_{F}$ are taken as $\left(N_{C}, N_{F}\right)=$ $(5,45)$, inspired by Eq. (6), and we have chosen $m_{F}=2.9 \times 10^{13}$ $\mathrm{GeV}$ as in the text. At the scale $m_{F}$ the dimensional transmutation takes place following the nonperturbative beta function $\beta_{g}^{\mathrm{NP}}$ in Eq. (7), or equivalently the Miransky scaling in Eq. (5), and the scalar potential is expected to be stabilized all the way above this scale

The Miransky scaling (the second relation in Eq. (5)) generates the nonperturtabutive scaling of $\alpha_{g}$ in the chiral broken phase (i.e. the nonperturbative scale anomaly) with respect to the cutoff $\Lambda=M_{\mathrm{pl}}[58]$ :

or $\quad \frac{\alpha_{g}(\mu)}{\alpha_{g}^{\mathrm{cr}}}=1+\frac{\pi^{2}}{\left[\log \frac{\mu}{m_{F}}\right]^{2}}, \quad$ for $\quad \alpha_{g}>\alpha_{g}^{\mathrm{cr}}$.

This nonperturbative running, which is actually "walking", governs the dynamics for $m_{F}<\mu<\Lambda=M_{\mathrm{pl}}$, with the characteristic large scale hierarchy, $m_{F} \ll \Lambda=M_{\mathrm{pl}}$. Note that $\alpha_{g}^{*}$ indeed plays the role of the AS fixed point in Eq. (7), and $m_{F}$ is the renormalization group invariant scale, like $\Lambda_{\mathrm{QCD}}$ in QCD, which signals the dimensional transmutation of the dynamical scale generation and is also the intrinsic scale characterizing the confining phase of the axicolor below $\mu=m_{F}$. In Fig. 1 we draw the running coupling behavior in the chiral broken phase, in the walking regime $\left(m_{F}<\mu<\right.$ $\left.\Lambda \equiv M_{\mathrm{pl}}\right)$.

For $\mu<2.9 \times 10^{13} \mathrm{GeV}$, we find $\left(\alpha_{\lambda_{1}}+\alpha_{\lambda_{2}}\right)<0$, which destabilizes the scalar potential at two-loop level. ${ }^{5}$ We may

\footnotetext{
5 The scalar sector itself can have a fictitious AS fixed point in the large $N_{F}$ limit at two-loop level. However, it is known on general grounds that scalar theories do not have fixed points at the weak coupling [59]. While fixed points might still exist at the strong coupling, the perturbation theory up to the four-loop order in the $\overline{\mathrm{MS}}$ scheme is unable to clarify [60]. Specifically, the conjectured fixed points at two loop
} 
thus identify the intrinsic scale $m_{F}$ as the threshold scale: $m_{F} \sim 2.9 \times 10^{13} \mathrm{GeV}$, which requires $\alpha_{g}$ to deviate from $\alpha_{g}^{*}$ by about $2 \%$ at $\mu=M_{\mathrm{pl}}$.

That is all about the central setup in the chiral broken phase, described in Fig. 1, from which the composite axion emerges and works for the relaxation mechanism for the strong $\mathrm{CP}$, as will be seen below.

\section{Identification of composite axion}

At $\mu=M_{\text {pl }}$ the chiral $U\left(N_{F}\right)_{L} \times U\left(N_{F}\right)_{R}$ symmetry is already broken down to $U\left(N_{F}\right)_{V}$, by the dynamical mass generation in Eq. (5) and developing nonzero chiral condensate $\langle-\bar{F} F\rangle$. However, axiclored fermions with mass $m_{F}$ do not decouple and still contribute to the renormalization-group running (walking) down until the intrinsic scale $m_{F}$, where the theory would be in the confining phase. At this scale, $N_{F}^{2}$ composite Nambu-Goldstone bosons are emergent. We shall momentarily disregard the $y$-Yukawa interaction in Eq. (3). Among those Nambu-Goldstone bosons, the chiral singlet one $\left(\sim \bar{F} i \gamma_{5} T^{0} F\right.$ associated with the singlet generator, $\left.T_{0}=1 / \sqrt{2 N_{F}} \cdot \mathbf{1}_{N_{F} \times N_{F}}\right)$ gets the mass of $\mathcal{O}\left(m_{F}\right)$, due to the axicolor instanton via the $U(1)_{F_{A}}$ anomaly, like $\eta^{\prime}$ in QCD. The colored Nambu-Goldstone bosons get masses of $\mathcal{O}\left(g_{s} /(4 \pi) m_{F}\right)$ by the QCD gluon interaction with the gauge coupling $g_{s}$, analogously to the photon exchange contribution to the charged pions in QCD. The $n_{F}^{2}$ QCD color-singlet Nambu-Goldstone bosons are at this point massless. Of those massless ones, we find the composite axion candidate, which is free from the axicolor $U(1)_{F_{A}}$ anomaly and coupled to the QCD topological operator $G_{\mu \nu} \tilde{G}^{\mu \nu}$ :

$a \sim \bar{F} i \gamma_{5} T_{a} F$,

with the generator

$T_{a} \equiv \sqrt{\frac{n_{F}}{6 N_{F}}}\left(\begin{array}{cc}-1_{3 \times 3} & 0 \\ 0 & \frac{3}{n_{F}} \cdot 1_{n_{F} \times n_{F}}\end{array}\right)$.

This composite-axion identification generalizes the one in the axicolor theory with smaller $N_{F}$ [31]. The associated chiral

Footnote 5 continued

located at $\left(\alpha_{\lambda_{1}}, \alpha_{\lambda_{2}}\right)=(0.333,-0.865)$ and $\left(\alpha_{\lambda_{1}}, \alpha_{\lambda_{2}}\right)=$ $(0.333,0.642)$ disappear into the complex plane at three loop, while at four loop, they re-appear at $\left(\alpha_{\lambda_{1}}, \alpha_{\lambda_{2}}\right)=(0.199,-0.736)$ and $\left(\alpha_{\lambda_{1}}, \alpha_{\lambda_{2}}\right)=(0.199,1.774)$. Results are not stable under the loop expansion, and couplings are not small enough to be under perturbative control. Therefore, the existence of a scalar fixed point cannot be trusted, hence the potential stability condition might not be reliable either. However, it will not essentially alter our main claim on the emergence of composite axion and the successful relaxation, which holds in the nonperturbative strong axicolor dynamics, without referring to the details of the scalar dynamics. symmetry, protecting the masslessness for $a$, is identified as the anomalous PQ symmetry, which is characterized by the chiral transformation with respect to $T_{a}$ in Eq. (9).

Thus, we have identified the composite axion and its associated PQ symmetry, among the emergent composite NambuGoldstone bosons and the spontaneously broken chiral symmetry. In the next section, we will discuss the mass and potential generation of the composite axion at the intrinsic scale $m_{F}$, by taking into account the $y$-Yukawa interaction in Eq. (3).

\section{Low-energy description below $m_{F}$}

Below $m_{F}$ all axi-hadrons would be decoupled, only leaving Nambu-Goldstone bosons (except $\eta^{\prime}$ like one). Then we work on a chiral Lagrangian in the chiral broken phase based on $G / H=S U\left(N_{F}\right)_{L} \times S U\left(N_{F}\right)_{R} / S U\left(N_{F}\right)_{V}$ coupled to the chiral scalar $M$ :

$\mathcal{L}=\frac{f^{2}}{4} \operatorname{tr}\left[D_{\mu} U^{\dagger} D^{\mu} U\right]+b f^{2} \operatorname{tr}\left[U^{\dagger} \mathcal{M}+\mathcal{M}^{\dagger} U\right]+\mathcal{L}_{\mathrm{WZW}}$,

with the suprion field $\mathcal{M}$ including the chiral scalar $M$ as

$\mathcal{M}=\left(\begin{array}{cc}0_{3 \times 3} & 0 \\ 0 & (M)_{n_{F} \times n_{F}}\end{array}\right)$.

Here $U$ is the chiral field parametrized by the NambuGoldstone boson fields $\pi$ as $U=e^{2 i \sum_{A=1}^{N_{F}^{2}-1} \frac{\pi^{A} T^{A}}{f}}$ with $T^{A}$ being $S U\left(N_{F}\right)$ generators and $f$ the axi-pion decay constant. This $U$ transforms under the chiral symmetry in the same way as $M$ does: $U \rightarrow g_{L} \cdot U \cdot g_{R}^{\dagger}\left(g_{L / R} \in\right.$ $\left.S U\left(N_{F}\right)_{L / R}\right)$. The covariant derivative acting on $U$ has been introduced by reflecting the partial-vectorlike gauging of the chiral symmetry as in Eq. (1), which takes the form $D_{\mu} U=\partial_{\mu} U-i\left[V_{\mu}, U\right]$, with the external gauge field $V_{\mu}=\left(\begin{array}{cc}g_{s}\left(G_{\mu}\right)_{3 \times 3} & 0 \\ 0 & 0_{n_{F} \times n_{F}}\end{array}\right)$, in which the QCD gluon fields $G_{\mu}$ are embedded. The last part $\mathcal{L}_{\text {WZW }}$ in Eq. (10) is the (covariantized) Wess-Zumino-Witten term $[61,62]$, which includes the QCD topological term, $G_{\mu \nu}^{\mathbf{a}} \tilde{G}^{\mathbf{a} \mu \nu}$ (with $\mathbf{a}=1, \ldots, 8)$, coupled to the composite axion $a$ in Eq. (8):

$$
\begin{aligned}
\mathcal{L}_{\text {WZW }} & \ni-\frac{N_{C}}{4 \pi^{2} f} \epsilon^{\mu \nu \rho \sigma} \operatorname{tr}\left[\partial_{\mu} V_{\nu} \partial_{\rho} V_{\sigma} \pi\right] \\
& \ni \frac{N_{C} g_{s}^{2}}{32 \pi^{2}} \frac{a}{f_{a}} G_{\mu \nu}^{\mathbf{a}} \tilde{G}^{\mathbf{a} \mu \nu},
\end{aligned}
$$

with the axion decay constant

$$
f_{a}=\sqrt{\frac{6 N_{F}}{n_{F}}} f .
$$


The coupling parameter $b$ in Eq. (10) can be fixed as follows: replace the $\psi$-fermion bilinear $\bar{\psi}_{R i} \psi_{L j}$ in Eq. (3) with a partial chiral field $u_{j i}$ as $\bar{\psi}_{R i} \psi_{L j} \approx\langle\bar{\psi} \psi\rangle \cdot u_{j i}=$ $\langle\bar{F} F\rangle \cdot u_{j i}$, where we have introduced the $S U\left(N_{F}\right)$ vectorial chiral condensate per flavor, $\langle\bar{\psi} \psi\rangle=\langle\bar{F} F\rangle$, which respects the axicolor-vectolike gauge dynamics via the Vafa-Witten's theorem [63]. Taking $u=1$ in the $b$ term in Eq. (10), and comparing them, we then find

$b=\frac{y\langle-\bar{F} F\rangle}{f^{2}}$.

Including the quartic-potential terms in Eq. (2), the potential of $M$ reads

$$
\begin{aligned}
V_{M}\left(\mu=m_{F}\right)= & -b f^{2} \operatorname{tr}\left[M+M^{\dagger}\right] \\
& +\lambda_{1} \operatorname{tr}\left[\left(M^{\dagger} M\right)^{2}\right]+\lambda_{2}\left(\operatorname{tr}\left[M^{\dagger} M\right]\right)^{2} .
\end{aligned}
$$

Thus the chiral condensate ( $b$ in Eq. (14)) generates the tadpole term for the chiral scalar $M$. Note that though the chiral condensate is generated already at $\mu=M_{\mathrm{pl}}$, the theory keeps (almost) scale-invariant down til $\mu \sim m_{F}$ due to the walking nature, as seen from the $\alpha_{g}$ running in Fig. 1. Thereby, the scalar potential in Eq. (15) has been defined at $\mu=m_{F}$, hence the tadpole term coefficient $\left(b f^{2}\right)$ should be read as $\left.\left(b f^{2}\right)\right|_{\mu=m_{F}}=\left.y\left(m_{F}\right)\langle-\bar{F} F\rangle\right|_{\mu=m_{F}}$, via Eq. (14).

Decomposing $M$ into scalar and pseudoscalar matrix parts as $M=S+i P$, the potential looks like

$V_{M}\left(m_{F}\right)=-\sqrt{2 n_{F}} b f^{2} \cdot S_{0}+\frac{1}{4}\left(\frac{\lambda_{1}}{n_{F}}+\lambda_{2}\right) \cdot S_{0}^{4}$,

where only the $S U\left(n_{F}\right)$-singlet scalar part $\left(S_{0} \cdot 1 / \sqrt{2 n_{F}}\right.$. $1_{n_{F} \times n_{F}}$ ) has survived, because others do not get the tadpole. We then see that the chiral scalar develops nonzero vacuum expectation value (VEV),

$$
\begin{aligned}
\langle M\rangle= & \left\langle S_{0}\right\rangle \cdot \frac{1}{\sqrt{2 n_{F}}} \cdot 1_{n_{F} \times n_{F}}=\left(\frac{b f^{2}}{2\left(\lambda_{1}+n_{F} \lambda_{2}\right)}\right)^{1 / 3} \\
& \cdot 1_{n_{F} \times n_{F}} \\
= & \left(\frac{n_{F} b f^{2}}{2(4 \pi)^{2}\left(\alpha_{\lambda_{1}}+\alpha_{\lambda_{2}}\right)}\right)^{1 / 3} \\
& \cdot 1_{n_{F} \times n_{F}} \equiv v_{M} \cdot 1_{n_{F} \times n_{F}},
\end{aligned}
$$

where we have replaced $\lambda_{1}$ and $\lambda_{2}$ with their fine structure constants, in Eq. (4).

Note that this VEV does not generate any extra NambuGoldstone bosons: turning off the Yuakwa coupling $y$ in Eq. (3) (as well as QCD charges), the theory possesses an enhanced global chiral $\left[U\left(N_{F}\right)_{L} \times U\left(N_{F}\right)_{R}\right] \times\left[U\left(n_{F}\right)_{L} \times\right.$ $\left.U\left(n_{F}\right)_{R}\right]$ symmetry. In this sense, nonzero $y$ explicitly breaks the enhanced chiral symmetry down to single $\left[U\left(n_{F}\right)_{L} \times\right.$
$\left.U\left(n_{F}\right)_{R}\right]$, which is spontaneously broken by the $F$-fermion chiral condensate in the axicolor sector. As seen above, the chiral condensate induces the tadpole $\operatorname{tr}\left[S_{0} \cdot 1_{n_{F} \times n_{F}}\right]$, which only respects the vectorial $U\left(n_{F}\right)_{F_{V}}$ symmetry, and it indeed explicitly breaks the original $U\left(n_{F}\right)$ chiral symmetry in the $M$ sector. Hence pseudoscalars in $M$ fully get massive by the $y$-induced tadpole, and acquire the same mass as the $\left(n_{F}-1\right)$-plet scalars, $m_{P}^{2}=\frac{2(4 \pi)^{2}}{n_{F}}\left(2 \alpha_{\lambda_{1}}+\alpha_{\lambda_{2}}\right) \cdot v_{M}^{2}$. Some of them also gets the mass from the axicolor and/or QCD axial anomalies, as will be clarified below. Thus no extra massless Nambu-Goldstone bosons emerge.

The chiral scalar VEV $\langle M\rangle$ backreacts on the $F$-fermion mass as the chiral explicit breaking effect, which in part includes the PQ symmetry breaking. We in fact see that the chiral Lagrangian in Eq. (10) gives the pseudoscalar potential terms:

$$
\begin{aligned}
V_{y}= & -b f^{2} \operatorname{tr}\left[U^{\dagger} \mathcal{M}+\text { h.c. }\right] \\
= & -b f^{2} \operatorname{tr}\left[U^{\dagger}\left(\begin{array}{cc}
0_{3 \times 3} & 0 \\
0 & (M)_{n_{F} \times n_{F}}
\end{array}\right)+\text { h.c. }\right] \\
= & -b f^{2} v_{M} \operatorname{tr}\left[e^{-2 i\left(\frac{\pi_{0}}{\sqrt{2 N_{F}} f}+3 \sqrt{\frac{n_{F}}{6 N_{F}}} \frac{\pi_{a}}{n_{F} f}\right)} \cdot 1_{n_{F} \times n_{F}}\right. \\
& \cdot e^{\left.2 i \frac{P_{0}}{\sqrt{2 n_{F}} v_{M}} \cdot 1_{n_{F} \times n_{F}}+\text { h.c. }\right]+ \text { others }} \\
= & -2 n_{F} b f^{2} v_{M} \cos \left(2 \frac{\pi_{0}}{\sqrt{2 N_{F}} f}+6 \frac{\pi_{a}}{n_{F} f_{a}}-\frac{2 P_{0}}{\sqrt{2 n_{F}} v_{M}}\right) \\
& + \text { others, }
\end{aligned}
$$

where we have parametrized $M$ as nonlinear realization, $M=v_{M} \cdot e^{2 i P / v_{M}}$ with $v_{M}$ in Eq. (17). The terms denoted by others do not contribute to the axicolor and QCD anomalies, hence can be taken to be zero in evaluating minimization of the potential. Then we find the potential term for $\pi_{0} \equiv \eta_{\mathrm{AC}}$ and $\pi_{a} \equiv a$ (normalized as $V_{y}\left(\eta_{\mathrm{AC}}=0, a=0, P_{0}=0\right)=$ $0)$,

$$
\begin{aligned}
V_{y}\left(\eta_{\mathrm{AC}}, a, P_{0}\right)= & 2 n_{F} b f^{2} v_{M}\left[1-\cos \left(2 \frac{\eta_{\mathrm{AC}}}{\sqrt{2 N_{F}} f}+\frac{6}{n_{F}} \frac{a}{f_{a}}\right.\right. \\
& \left.\left.-\frac{2 P_{0}}{\sqrt{2 n_{F}} v_{M}}\right)\right] .
\end{aligned}
$$

As shown in Appendix A, no phase parameter arises from the axicolor sector including the $y$-Yukawa term, due to masslessness of QCD-colored fermions, hence no shift ambiguity in the argument of cosine is present in Eq. (19). Note that the potential terms in Eq. (19) necessarily come with the suppression factor of $1 / n_{F}$ or $1 / N_{F}$. Thus, the $y$-Yukawa interaction generates the potential terms for the axion as well as the mixing partners $\eta_{\mathrm{AC}}$ and $P_{0}$, as the subleading-order effect in the anti-Veneziano limit including the large $N_{F}$ expansion. 


\section{Relaxation and axion mass}

In addition to the potential terms in Eq. (19), the quantum anomalies give rise to the corrections to the pseudoscalar potentials. From the axicolor sector (with the axicolor gauge field $\mathcal{G}$ with the gauge coupling $g_{\mathrm{AC}}$ ), we have

$\mathcal{L}_{\mathcal{G} \tilde{\mathcal{G}}}=\frac{g_{\mathrm{AC}}^{2}}{32 \pi^{2}}\left(\frac{\eta_{\mathrm{AC}}}{\sqrt{2 N_{F}} f}+\frac{P_{0}}{\sqrt{2 n_{f}} v_{M}}\right) \mathcal{G}_{\mu \nu}^{A} \tilde{\mathcal{G}}^{\mu \nu A}$

The axicolor instanton therefore yields the potential,

$$
V_{\mathcal{G} \tilde{\mathcal{G}}}^{\text {instanton }} \sim m_{F}^{4}\left[1-\cos \left(\frac{\eta_{\mathrm{AC}}}{\sqrt{2 N_{F}} f}+\frac{P_{0}}{\sqrt{2 n_{f}} v_{M}}\right)\right] .
$$

Again, the potential terms arise as the subleading effect in the large $N_{F}$ expansion, suppressed by $1 / n_{F}$ or $1 / N_{F}$.

Besides, the QCD sector develops the anomaly along with the strong $\mathrm{CP}$ violating parameter $\theta$,

$\mathcal{L}_{G \tilde{G}}=\frac{g_{s}^{2}}{32 \pi^{2}}\left(N_{C} \frac{a}{f_{a}}+N_{C} \frac{\eta_{\mathrm{AC}}}{\sqrt{2 N_{F}} f}+\theta\right) G_{\mu \nu}^{\mathbf{a}} \tilde{G}^{\mathbf{a} \mu \nu}$,

with which the QCD instanton generates the potential terms,

$$
\begin{aligned}
& V_{G \tilde{G}}^{\text {instanton }} \sim\left(\Lambda_{\mathrm{QCD}}^{\text {flavor-singlet }}\right)^{4}[1 \\
& \left.\quad-\cos \left(N_{C} \frac{a}{f_{a}}+N_{C} \frac{\eta_{\mathrm{AC}}}{\sqrt{2 N_{F}} f}+\theta\right)\right] .
\end{aligned}
$$

The overall scale $\Lambda_{\mathrm{QCD}}^{\text {flavor-singlet }}$ denotes a flavor-singlet part of the QCD instanton contribution, such as $\sim m_{u} m_{d} \Lambda_{\mathrm{QCD}}^{2}$ for the lightest two quark flavors, arising from the flavorsinglet nature of the QCD vacuum (See, e.g., a review [3]). ${ }^{6}$ The $\eta_{\mathrm{AC}}$ terms are suppressed by factors of $1 / N_{F}$, while the axion terms survive even in the leading order of the large $N_{F}$ expansion. This is due to the fact that the axion coupling to $G_{\mu \nu} \tilde{G}^{\mu \nu}$ arises from the QCD-charged $\psi^{\alpha}$ fermion loop which does not come with the $\left(1 / n_{F}\right)$ factor (See Eqs. (12) with Eq. (9)). Note that even in terms of the anti-Veneziano limit $\left(N_{C}, N_{F} \gg 1, N_{F} / N_{C} \ll 1\right)$, only the axion term together with the QCD $\theta$ parameter survives.

\footnotetext{
$\overline{6 \text { The QCD } \eta^{\prime}}$ would mix there, as discussed in [3], but would yield the mass from another instanton-induced potential energy $\sim \Lambda_{\mathrm{OCD}}^{4}$, irrespective to the flavor-singlet condition. Incorporation of the QCD $\eta^{\prime}$ into the minimization of the full potential would not substantially modify Eqs. (24) and (26).
}

Combining those with Eq. (19), we find the full potential to be minimized by the following condition:

$$
\begin{aligned}
& \left(\frac{\eta_{\mathrm{AC}}}{\sqrt{2 N_{F}} f}+\frac{P_{0}}{\sqrt{2 n_{f}} v_{M}}\right)=\left(N_{C} \frac{a}{f_{a}}+N_{C} \frac{\eta_{\mathrm{AC}}}{\sqrt{2 N_{F}} f}+\theta\right) \\
& =\left(2 \frac{\eta_{\mathrm{AC}}}{\sqrt{2 N_{F}} f}+6 \frac{a}{n_{F} f_{a}}-\frac{2 P_{0}}{\sqrt{2 n_{F}} v_{M}}\right)=0 .
\end{aligned}
$$

In the anti-Veneziano limit, i.e., $N_{F} \gg 1, N_{C} \gg 1$ and $N_{F} / N_{C} \gg 1$, only the $a-G-\tilde{G}$ coupling is left so that the minimization condition is simplified to

$\frac{a}{f_{a}} \stackrel{N_{F} \gg 1}{\approx}-\frac{\theta}{N_{C}}$

This is precisely the limit for the (composite) QCD axion feeding the mass only through the QCD instanton effect, which is essentially identical to the one in the original axicolor scenario $[30,31]$ without Yukawa couplings as in Eq. (3). However, this case is too extreme to be phenomenologically viable, because all the $y$-Yukawa term contributions to the potential are suppressed by $1 / N_{F}$, leaving a number of exactly massless Nambu-Goldstone bosons.

By exactly solving Eq. (24), the relaxation of the $\theta$ parameter reads

$$
\begin{aligned}
\frac{a}{f_{a}} & =-\frac{\theta}{N_{C}\left(1-\frac{3}{2 n_{F}}\right)}, \\
\frac{\eta_{\mathrm{AC}}}{\sqrt{2 N_{F}} f} & =-\frac{3}{2} \frac{a}{f_{a} n_{F}}, \\
\frac{P_{0}}{\sqrt{2 n_{F}} v_{M}} & =-\frac{\eta_{\mathrm{AC}}}{\sqrt{2 N_{F}} f} .
\end{aligned}
$$

This relaxation works including heavier pseudoscalar background fields other than the composite axion, which is called ultraviolet solutions as recently discussed in the literature [36-39]. We will come back to this point again later. Thus, the viable composite axion with the successful relaxation mechanism arises as the subleading effect in the large $N_{F}$ expansion, i.e., the subleading correction to the anti-Veneziano limit.

At $\mu=M_{\mathrm{pl}}$, the chiral symmetry of the axicolored fermions is only broken in part by the $y$-Yuakwa interaction with the chiral scalar $M$. No higher dimensional interactions, including gravitational chiral breaking, are allowed by the quantum scale invariance at this Planck scale, robustly protected by the AS fixed point. The partially gauged perturbative QCD interactions cannot induce any chiral breaking at any loop level, because it is protected by the chiral symmetry.

The $y$-Yukawa interaction will not generate any chiral breaking, as long as the Yukawa coupling is perturbative enough, as we have assumed (e.g. $10^{-90} \lesssim \alpha_{y}\left(m_{F}\right) \lesssim 10^{-6}$, 
for a later reference). Thus the $y$-Yukawa interaction will be frozen at $m_{F}$ because of decoupling of axicolor fermions,

Even over the Planck scale, the present scalegenesis can be protected by an asymptotic safety quantum gravity with the nontrivial ultraviolet fixed point [64-79]. Thereby, any quantum gravity contribution to the chiral symmetry breaking, arising as higher dimensional operators suppressed by the Planck scale, could be absent as well (See also footnote 3 ). Thus the theory could keep (nearly) scale invariant all the way in running ("walking") down until the axicolor undergoes the dimensional transmutation at $m_{F}$, due to the walking nature in the chiral broken phase along with the Miransky scaling (Eq. (5) or Eq. (7)). This implies that the theory could have no quality problem [10-13].

Though being frozen, the remnant of $y$-Yuakwa interaction will be crucial only after the scale generation at $m_{F}$, beause it will trigger the chiral scalar VEV, and feed back to the axicolored fermion masses. This develops the composite axion potential as in Eq. (19). Actually, this phenomenon arises as a subleading effect in terms of the present asymptotic safety (with the large $N_{F}$ and $N_{C}$ ), as noted above. So, the axion (and other pseudo Nambu-Goldstone bosons) will be well under control by perturbation around the antiVeneziano limit, as well as the standard chiral perturbation theory as in the case of QCD pion and/or axion. Thus the $y$ - Yukawa induced axion potential form in Eq. (19) will be intact, as long as the chiral and the AS protections work well.

Moreover, of interest is to note that the axicolor-theta term is unphysical in the present setup, because QCD-colored $\psi^{\alpha}$ fermions do not couple to the chiral scalar $M$, and do not get the mass directly by the $y$ coupling. Thus the axicolortheta term can completely be rotated away by the residual chiral symmetry. Hence the potential form of Eq. (19) is unambiguous under the axial rotation in the axicolor sector, as it stands. More details are provided in Appendix A.

Thus the present relaxation mechanism for the QCD strong CP phase perfectly works, supported by the asymptotic safety (large $N_{F}$ walking), which is already fulfilled at the infrared dynamical scale $m_{F}$, much higher than the QCD scale. Since the QCD-axicolored fermions can also couple to electroweak gauge bosons at some higher loop level, the theta parameter in the axicolor sector would actually be not exactly vanishing, through the renormalization evolution arising from the standard model alone. Though this would shift the $\mathrm{CP}$ conserving vacuum, the induced $\mathrm{CP}$ violation should be much highly suppressed compared to the renormalization effect on the theta parameter within the standard model, which is even negligibly tiny. Thus, the strong CP problem is practically and essentially solved at such an ultraviolet scale by a hybrid relaxation mechanism involving other heavier psuedoscalars, as in Eq. (26). Similar ultraviolet solutions in a context of composite axions have been discussed in the literature [36-39].
By integrating out heavier pseudoscalars $\left(\eta_{\mathrm{AC}}\right.$ and $\left.P_{0}\right)$ with mass predominantly fed by the axicolor instanton effect, the total axion mass (squared) reads

$$
\begin{aligned}
m_{a}^{2} \simeq & \frac{\left(\Lambda_{\mathrm{QCD}}^{\text {flavor-singlet }}\right)^{4}+\frac{72}{n_{F}} \cdot m_{F 0}\langle-\bar{F} F\rangle}{f_{a}^{2}} \\
& \equiv\left(m_{a}\right)_{\mathrm{QCD}}^{2}+\left(m_{a}\right)_{\text {axicolor }}^{2},
\end{aligned}
$$

where $m_{F 0} \equiv y v_{M}$. Now we discuss the generic property of $\left(m_{a}\right)_{\text {axicolor }}$ in the anti-Veneziano asymptotic safety. To this end, note first that the axicolor-sector vacuum-energy part, $m_{F 0}\langle-\bar{F} F\rangle$ in Eq. (27) is renormalization group invariant. We set the reference renormalization scale $\mu$ to $m_{F}$. Then we can generically evaluate the chiral condensate per flavor (renormalized at $\mu=m_{F}$ ) and the axi-pion decay constant $f$ (and $f_{a}$ through Eq. (13)), as a function of $m_{F}$ :

$$
\begin{aligned}
\langle-\bar{F} F\rangle_{\mu=m_{F}} & =\frac{N_{C}}{4 \pi^{2}} \kappa_{C} m_{F}^{3}, \\
f^{2} & =\frac{N_{C}}{4 \pi^{2}} \kappa_{F} m_{F}^{2},
\end{aligned}
$$

up to constants $\kappa_{F}$ and $\kappa_{C}$ which can be fixed by solving the walking dynamics in the chiral broken phase. When we use the ladder Schwinger-Dyson equation along with the PagelsStorkar formula [80], these $\kappa_{F}$ and $\kappa_{C}$ can be estimated to be $[35,81]$

$\kappa_{F} \simeq 2 \xi^{2} \simeq 2.4$

$\kappa_{C} \simeq \frac{32 \xi^{2}}{\pi^{2}} \simeq 3.9$,

where $\xi \simeq 1.1$. Then a reference value of the axion decay constant $f_{a}$ in Eq. (13) is estimated by taking account the aforementioned stability bound for the scalar potential in the walking regime $\left(m_{F} \sim 2.9 \times 10^{13} \mathrm{GeV}\right)$ as $f_{a} \sim 10^{14} \mathrm{GeV}$, for $N_{F}=9 N_{C}=45$.

Using Eq. (28) together with Eq. (17) and replacing $y$ with its fine structure constant $\alpha_{y}$ in Eq. (4), we express the axicolor-induced axion mass in Eq. (27) by making the $N_{C}$ and $N_{F}$ dependence manifest:

$$
\begin{aligned}
\left(\frac{\left(m_{a}\right)_{\text {axicolor }}}{m_{F}}\right)^{2} & =\frac{12}{N_{F}}\left(\frac{\kappa_{C}}{\kappa_{F}}\right) \cdot\left(\frac{\left[m_{F 0}\right]_{\mu=m_{F}}}{m_{F}}\right) \\
& =\frac{12}{N_{F}}\left(\frac{\kappa_{C}}{\kappa_{F}}\right) \cdot\left[\frac{2 n_{F} \alpha_{y}^{2}}{N_{C}\left(\alpha_{\lambda_{1}}+\alpha_{\lambda_{2}}\right)}\right]_{\mu=m_{F}}^{1 / 3} .
\end{aligned}
$$

Note that $m_{F}$ does not scale with $N_{C}$ or $N_{F}$. Thus $\left(m_{a}\right)_{\text {axicolor }}$ is potentially a subleading term of $\mathcal{O}\left(1 / \sqrt{N_{F}}\right)$ and becomes 
vanishingly small in the anti-Veneziano limit,

$$
\begin{array}{ll}
\left(m_{a}\right)_{\text {axicolor }} \rightarrow 0, \quad \text { i.e., } \quad m_{a} \rightarrow\left(m_{a}\right)_{\mathrm{QCD}}, \\
\text { as } \quad N_{F}, N_{C} \rightarrow \infty, \quad \text { with } \quad N_{F} / N_{C} \gg 1,
\end{array}
$$

in accordance with the same relaxation form as the QCD axion's, in Eq. (25). This feature is contrast to the $\eta^{\prime}$ like mass in the anti-Veneziano limit, which goes like $\gg m_{F}$, as was discussed in the literature [35].

With Eq. (30) for finite $N_{F}$ and $N_{C}$ (e.g. $N_{F}=9 N_{C}=$ 45 ), the reference values may be in a range involving $m_{a} \sim$ $\left(m_{a}\right)_{\mathrm{QCD}} \sim 10^{-7} \mathrm{eV}$ for $\alpha_{y}\left(m_{F}\right) \sim 10^{-90}$, and $m_{a} \sim 10^{12}$ $\mathrm{GeV}$ for $\alpha_{y}\left(m_{F}\right) \sim 10^{-6}$. The former is the $\mathrm{QCD}$ axion limit with $f_{a} \sim 10^{14} \mathrm{GeV}$, while the latter is just a conservative upper bound for the current renormalization group analysis neglecting $\alpha_{y}$ compared to the perturbative scalar quartic couplings which we take $\left.\left(\alpha_{\lambda_{1}}+\alpha_{\lambda_{2}}\right)\right|_{\mu=m_{F}} \simeq 1.1 \times 10^{-5}$ from the two-loop level analysis. ${ }^{7}$ In the QCD axion limit, the composite axion with $f_{a} \sim 10^{14} \mathrm{GeV}$ is currently in an unexplored window [82].

\section{Conclusion and discussions}

In this paper, we proposed a new class of a composite axion providing a solution to the strong $\mathrm{CP}$ problem, which simultaneously resolves the hierarchy and triviality problems potentially existing in the PQ symmetry breaking scenario. The composite axion arises in a dynamical scalegenesis, and shows up in a subleading domain of an "anti-Veneziano" limit of a confining gauge theory $\left(N_{F}, N_{C} \rightarrow \infty\right.$, with $N_{F} / N_{C} \gg 1$ ) having an AS fixed point. The confining gauge theory, what we call axicolor, couples to $N_{F}$ fermions, three of which are gauged by the QCD charge and the rest $n_{F}\left(=N_{F}-3 \gg 1\right)$ fermions couple to $n_{F}^{2}$ scalars to ensure the chiral symmetry for $n_{F}$ fermions by forming the Yukawa coupling in a scale invariant way.

We addressed that in the strong-gauge coupling regime, the large $N_{F}$ axicolor theory is almost governed by a strongQED like dynamics with the constant gauge coupling identified as the AS coupling, that is a walking dynamics. The full chiral symmetry for $N_{F}$ axicolored fermions, as well as the scale symmetry, are spontaneously broken by the dynamical fermion mass generation following the Miransky scaling (Eq. (5)). This chiral breaking generates an axion-like pseudoscalar composite and the Yukawa coupling with the developed scalar VEV serves as the axion mass, as well as the other pseudo Nambu-Goldstone boson's.

\footnotetext{
7 The two-loop perturbative running couplings with this reference value of $\left.\left(\alpha_{\lambda_{1}}+\alpha_{\lambda_{2}}\right)\right|_{\mu=m_{F}}$ grows up to higher scales, until the fixed point $\left(\alpha_{\lambda_{1}}, \alpha_{\lambda_{2}}\right)=(0.333,0.642)$, hence no destabilization of the scalar potential is present all the way during the running.
}

It was shown that in the exact anti-Veneziano limit, the composite axion becomes the QCD axion, and the relaxation of the QCD theta parameter works in the same way as in the QCD axion's (Eqs. (25) and (31)). The subleading nature of the anti-Veneziano limit makes the scenario more phenomenologically viable, and yields a heavy composite axion (Eq. (30)).

The present relaxation of the strong $\mathrm{CP}$ phase is so-called the ultraviolet solution, along with a couple of heavy (composite) pseudoscalars, as well as the composite axion (Eq. (26)). This is now interpreted as the subleading phenomenon of the anti-Veneziano-AS axicolor theory, and is robustly established due to the (almost) scale invariance during the walking regime (Fig. 1).

In closing, we give several comments on the directions along the presently proposed scenario, which deserve to be pursued as the future works:

- The present gauge-Yukawa-scalar theory can be projected up to the gauged Nambu-Jona Lasinio theory, as has been noted in $[83,84]$. In that case, the chiral scalar $M$ would be regarded as axicolored fermion bound states generated by the strong gauge and four-fermion interactions [84]. This gauged Nambu-Jona Lasinio theory can then be almost scale-invariant until reaching the ultraviolet fixed point (along the "fixed line" in the gauge and four-fermion coupling space [85-92]). It would be worth revisiting the gauged Nambu-Jona Lasinio theory in light of the emergence of the composite axion. In particular, in that case all the factors relevant to the axion mass formula in Eq. (30) can be computed, so it would be possible to estimate more definite referenced value of $m_{a} / m_{F}$.

- We have presently focused only on the asymptotically safe dynamical scalegenesis for the PQ sector. Extension of this scenario to the electroweak sector, i.e., the scalegenesis of the electroweak scale, might be straightforward by simply introducing the (currently suppressed) Higgs-portal coupling, like $\left(H^{\dagger} H\right)\left(\operatorname{tr}\left[M^{\dagger} M\right]\right)$. In that case, however, the size of such a portal coupling needs to be taken to be so tiny, which would revive the gaugehierarchy problem. One possible way out is to address the dynamical origin of the chiral scalar $M$, as commented in the first bullet above, that is replacing the present linearsigma model-like description for the chiral scalar $M$ by the gauged Nambu-Jona Lasinio theory. The Higgs portal coupling is then absent at the leading order of the large $n_{F}$ expansion, because the QCD-singlet axicolored fermions do not couple to $H^{\dagger} H$ at this level. Including the standard-model contribution as the subleading order of the large $n_{F}$, the portal coupling could be generated as small violation of the scale invariance. It would be highly suppressed and generated below the axicolor scale $m_{F}$, by involving the SM quark and gluon loops at higher loop 
levels of perturbation in the SM with the chiral scalar $M$. Taking into account the renormalization evolution of the induced portal coupling, scaling down to the electroweak scale from the axicolor scale $m_{F}$, might realize a desired size of the negative-squared Higgs mass parameter necessary to generate the Higgs VEV at the electroweak scale. This issue is noteworthy, to be pursued in details elsewhere.

- The composite axion with large $N_{C}$ generically suffers from the domain wall problem. However, this is avoidable if an inflation takes place after the dynamical chiral symmetry breaking triggered by the axicolor (and four-fermion) dynamics. The inflaton can be played by a composite dilaton, arising as a pseudo NambuGoldstone boson associated with the spontaneous scale symmetry breaking due to the walking (i.e. nearly scale invariant) dynamics of the axicolor, hence is expected to be light enough to present in the low-energy dynamics together with the axi-pions and composite axion. As has been shown in [93], the walking dynamics can naturally achieve a small field inflation with the light walking dilaton as the inflaton. Or, as was discussed in [94-96], in the framework of the gauged Nambu-Jona Lasinio theory which could be dual to the gauge-Yukawa-scalar theory, allowing a nonminimal coupling to gravity can achieve a large field inflation, which was shown to favor somewhat a large $N_{F}$. For both cases, it would be interesting to examine how the composite axion can contribute to ending the inflation, i.e., so-called the waterfall scenario $[97,98]$.

- Axicolor hadrons as well as the chiral scalars are heavy enough to be decoupled at low energy, except the lighter composite pseudo Nambu-Goldstone bosons (including a possibly light composite dilaton). Other than the composite axion, those pseudoscalars get the mass on the same order as $m_{a}$, arising at the subleading order of the large $N_{F}$ limit. They would be dark matters, and can be thermally produced by axi-hadronic interactions, such as the axi-baryon annihilations and/or $3-2$ processes like dark pions (strongly interacting massive particle, SIMP) [99-101]. More on this dark matter physics, in association with the composite axion, would be worth to pursue.

- For the present proposal to be more realistic, it needs more rigorous discussion on certain existence of asymptotic safe confining gauge-Yukawa-scalar theory in the nonperturbative regime, by using the functional renormalization group [102-105] and lattice simulations as the pioneering work in [57].

Acknowledgements S.M. thanks Jie Liu for useful discussions. S.M. work was supported in part by the National Science Foundation of China (NSFC) under Grant No.11747308, 11975108, 12047569 and the Seeds Funding of Jilin University. The work of H.I. was partially supported by JSPS KAKENHI Grant Number 18H03708.
Data Availability Statement This manuscript has no associated data or the data will not be deposited. [Authors' comment: There are no associated data available.]

Open Access This article is licensed under a Creative Commons Attribution 4.0 International License, which permits use, sharing, adaptation, distribution and reproduction in any medium or format, as long as you give appropriate credit to the original author(s) and the source, provide a link to the Creative Commons licence, and indicate if changes were made. The images or other third party material in this article are included in the article's Creative Commons licence, unless indicated otherwise in a credit line to the material. If material is not included in the article's Creative Commons licence and your intended use is not permitted by statutory regulation or exceeds the permitted use, you will need to obtain permission directly from the copyright holder. To view a copy of this licence, visit http://creativecomm ons.org/licenses/by/4.0/.

Funded by $\mathrm{SCOAP}^{3}$.

\section{A Massless fermion solution for axicolor CP violation}

This appendix shows that the axicolor sector is free from $\mathrm{CP}$ violating-phases due to the flavor-singlet nature of the axicolor.

We focus on the anomalous sector:

$$
\begin{aligned}
& \mathcal{L}_{U(1)_{F_{A}}, U(1)_{\mathrm{PQ}}}^{\text {anomalous }}=-\sum_{i=1}^{N_{F}}\left|m_{i}\right|\left[e^{1 \theta_{y}} \bar{F}_{L i} F_{R i}+\text { h.c. }\right] \\
& +\frac{\theta_{\text {axicolor }}}{32 \pi^{2}} \mathcal{G}_{\mu \nu} \tilde{\mathcal{G}}^{\mu \nu}+\frac{\theta_{\mathrm{QCD}}}{32 \pi^{2}} G_{\mu \nu} \tilde{G}^{\mu \nu}
\end{aligned}
$$

where $\theta_{\mathrm{QCD}}=\theta-\operatorname{argdet}\left[m_{q}\right]$ with the QCD theta parameter $\theta$ and complex quark mass matrix $m_{q}, m_{1}=m_{2}=m_{3}=0$ and $m_{4}=\cdots=m_{N_{F}}=m$. First of all, we note that the phase of the $y$-Yukawa coupling can be removed by field redefinition of $F_{L}$ and $F_{R}$, like $F_{L} \rightarrow e^{i \theta_{y}} F_{L}$ and $F_{R} \rightarrow e^{i \theta_{y}} F_{R}$, which keeps the other terms in the theory being invariant. Therefore we start with the phase-less Yukawa term:

$$
\begin{gathered}
\mathcal{L}_{U(1)_{F_{A}}, U(1) \mathrm{PQ}}^{\text {anomalous }}=-\sum_{i=1}^{N_{F}}\left|m_{i}\right|\left[\bar{F}_{L i} F_{R i}+\text { h.c. }\right] \\
+\frac{\theta_{\text {axicolor }}}{32 \pi^{2}} \mathcal{G}_{\mu \nu} \tilde{\mathcal{G}}^{\mu \nu}+\frac{\theta_{\mathrm{QCD}}}{32 \pi^{2}} G_{\mu \nu} \tilde{G}^{\mu \nu} .
\end{gathered}
$$

Under the $U(1)_{F_{A}}$ and $U(1)_{\mathrm{PQ}}$ rotations, the $F$-axicolored fermion fields transform as

$$
\begin{aligned}
F_{i} & \rightarrow\left[e^{-i \gamma_{5} \alpha_{0} T_{0} / 2}\right]_{i j} \cdot F_{j} \quad \text { with } \\
T_{0} & =\frac{1}{\sqrt{2 N_{F}}} \operatorname{diag}\{1, \ldots, 1\}, \\
F_{i} & \rightarrow\left[e^{-i \gamma_{5} \alpha_{a} T_{a} / 2}\right]_{i j} \cdot F_{j} \quad \text { with } \\
T_{a} & =\sqrt{\frac{n_{F}}{6 N_{F}}} \operatorname{diag}\left\{-1,-1,-1,3 / n_{F}, \ldots, 3 / n_{F}\right\} .
\end{aligned}
$$


It is convenient to decompose $F$ into the QCD-charged triplet $(Q)$ and non-charged ones $(\chi)$, so that the $U(1)_{F_{A}}$ and $U(1)_{P Q}$ transformations above take the form

$$
\begin{aligned}
F_{i} & =\left(\begin{array}{l}
Q \\
\chi
\end{array}\right) \rightarrow\left(\begin{array}{l}
e^{-i \gamma_{5} \alpha_{F_{A}} / 2} Q \\
e^{-i \gamma_{5} \alpha_{F_{A}} / 2} \chi
\end{array}\right) \quad \text { with } \quad \alpha_{F_{A}}=\sqrt{\frac{1}{2 N_{F}}} \alpha_{0}, \\
F_{i} & =\left(\begin{array}{l}
Q \\
\chi
\end{array}\right) \rightarrow\left(\begin{array}{l}
e^{-i \gamma_{5} \alpha_{Q} / 2} Q \\
e^{-i \gamma_{5} \alpha_{\chi} / 2} \chi
\end{array}\right) \quad \text { with } \alpha_{Q}=-\sqrt{\frac{n_{F}}{6 N_{F}}} \alpha_{a} \\
& =-\frac{n_{F}}{3} \alpha_{\chi} .
\end{aligned}
$$

These anomalous $U(1)_{F_{A}}$ and $U(1)_{\mathrm{PQ}}$ rotations transform the Lagrangian like

$$
\begin{aligned}
& \mathcal{L}_{U(1)_{F_{A}}, U(1)_{\mathrm{PQ}}}^{\text {anomalous }} \rightarrow-\left|m_{Q}\right| e^{-i\left(\alpha_{F_{A}}+\alpha_{Q}\right)} \bar{Q}_{L} Q_{R} \\
& -\left|m_{\chi}\right| e^{-i\left(\alpha_{F_{A}}+\alpha_{\chi}\right)} \bar{\chi}_{L} \chi_{R}+\text { h.c. } \\
& +\frac{\theta_{\text {axicolor }}-N_{F} \alpha_{F_{A}}}{32 \pi^{2}} \mathcal{G}_{\mu \nu} \tilde{\mathcal{G}}^{\mu \nu} \\
& +\frac{\theta_{\mathrm{QCD}}-N_{C}\left(\alpha_{Q}+\alpha_{F_{A}}\right)}{32 \pi^{2}} G_{\mu \nu} \tilde{G}^{\mu \nu}
\end{aligned}
$$

where $m_{Q}=m_{1,2,3}=0$ and $m_{\chi}=m_{4, \ldots, N_{F}}=m$.

Choosing the $\alpha_{F_{A}}$ phase convention,

$\alpha_{F_{A}}=\frac{\theta_{\text {axicolor }}}{N_{F}}$,

we may remove the axicolor-topological term. Since the axicolor interaction is flavor-blind, we require the flavor singlet condition for the phase dependent interaction terms, just like the QCD case $[106,107]$. For infinitesimal phases, we thus have

$\left|m_{Q}\right|\left(\alpha_{F_{A}}+\alpha_{Q}\right)=\left|m_{\chi}\right|\left(\alpha_{F_{A}}+\alpha_{\chi}\right) \equiv x$.

Note here that $m_{Q}=0$, hence $x=0$. which constrains the $\mathrm{PQ}$ and $U(1)_{F_{A}}$ phases as

$\alpha_{F_{A}}+\alpha_{\chi}=0$

leaving an arbitrary phase parameter combination,

$\alpha_{F_{A}}+\alpha_{Q}=$ arbitrary.

Thus, the axicolored sector is completely $\mathrm{CP}$ invariant at this point, due to the massless $Q$ fermions, where the original $\theta_{\text {axicolor }}$ is rotated away by the $U(1)_{F_{A}}$ transformation with $\alpha_{0}=\sqrt{2 / N_{F}} \theta_{\text {axicolor }}$ and the PQ transformation with $\alpha_{a}=$ $-\sqrt{2 n_{F} /\left(3 N_{F}\right)} \theta_{\text {axicolor }}$.

\section{References}

1. R.D. Peccei, H.R. Quinn, Phys. Rev. D 16, 1791-1797 (1977). https://doi.org/10.1103/PhysRevD.16.1791

2. M.A. Shifman, A.I. Vainshtein, V.I. Zakharov, Nucl. Phys. B 166, 493-506 (1980). https://doi.org/10.1016/0550-3213(80)90209-6

3. J.E. Kim, G. Carosi, Rev. Mod. Phys. 82 (2010), 557-602 [erratum: Rev. Mod. Phys. 91 (2019) no.4, 049902] https://doi.org/10. 1103/RevModPhys.82.557. arXiv:0807.3125 [hep-ph]

4. R.D. Peccei, H.R. Quinn, Phys. Rev. Lett. 38, 1440-1443 (1977). https://doi.org/10.1103/PhysRevLett.38.1440

5. S. Weinberg, Phys. Rev. Lett. 40, 223-226 (1978). https://doi.org/ 10.1103/PhysRevLett.40.223

6. F. Wilczek, Phys. Rev. Lett. 40, 279-282 (1978). https://doi.org/ 10.1103/PhysRevLett.40.279

7. J.E. Kim, Phys. Rev. Lett. 43, 103 (1979). https://doi.org/10.1103/ PhysRevLett.43.103

8. A.R. Zhitnitsky, Sov. J. Nucl. Phys. 31, 260 (1980)

9. M. Dine, W. Fischler, M. Srednicki, Phys. Lett. B 104, 199-202 (1981). https://doi.org/10.1016/0370-2693(81)90590-6

10. R. Holman, S.D.H. Hsu, T.W. Kephart, E.W. Kolb, R. Watkins, L.M. Widrow, Phys. Lett. B 282, 132-136 (1992). https:// doi.org/10.1016/0370-2693(92)90491-L arXiv:hep-ph/9203206 [hep-ph]

11. M. Kamionkowski, J. March-Russell, Phys. Lett. B 282, 137141 (1992). https://doi.org/10.1016/0370-2693(92)90492-M arXiv:hep-th/9202003 [hep-th]

12. S.M. Barr, D. Seckel, Phys. Rev. D 46, 539-549 (1992). https:// doi.org/10.1103/PhysRevD.46.539

13. S. Ghigna, M. Lusignoli, M. Roncadelli, Phys. Lett. B 283, 278281 (1992). https://doi.org/10.1016/0370-2693(92)90019-Z

14. E.J. Chun, A. Lukas, Phys. Lett. B 297, 298-304 (1992). https:// doi.org/10.1016/0370-2693(92)91266-C arXiv:hep-ph/9209208 [hep-ph]

15. M. Bastero-Gil, S.F. King, Phys. Lett. B 423, 27-34 (1998). https://doi.org/10.1016/S0370-2693(98)00124-5 arXiv:hep-ph/9709502 [hep-ph]

16. K.S. Babu, I. Gogoladze, K. Wang, Phys. Lett. B 560, 214 222 (2003). https://doi.org/10.1016/S0370-2693(03)00411-8 arXiv:hep-ph/0212339 [hep-ph]

17. H. Fukuda, M. Ibe, M. Suzuki, T.T. Yanagida, Phys. Lett. B 771, 327-331 (2017). https://doi.org/10.1016/j.physletb.2017.05.071 arXiv:1703.01112 [hep-ph]

18. M. Duerr, K. Schmidt-Hoberg, J. Unwin, Phys. Lett. B 780, 553-556 (2018). https://doi.org/10.1016/j.physletb.2018.03.054 arXiv:1712.01841 [hep-ph]

19. Q. Bonnefoy, E. Dudas, S. Pokorski, Eur. Phys. J. C 79(1), 31 (2019). https://doi.org/10.1140/epjc/s10052-018-6528-z. arXiv:1804.01112 [hep-ph]

20. M. Redi, R. Sato, JHEP 05, 104 (2016). https://doi.org/10.1007/ JHEP05(2016)104 arXiv:1602.05427 [hep-ph]

21. L. Darmé, E. Nardi, Phys. Rev. D 104(5), 055013 (2021). https:// doi.org/10.1103/PhysRevD.104.055013. arXiv:2102.05055 [hep-ph]

22. Y. Nakai, M. Suzuki, Phys. Lett. B 816, 136239 (2021). https://doi. org/10.1016/j.physletb.2021.136239 arXiv:2102.01329 [hep-ph]

23. L. Randall, Phys. Lett. B 284, 77-80 (1992). https://doi.org/10. 1016/0370-2693(92)91928-3

24. L. Di Luzio, E. Nardi, L. Ubaldi, Phys. Rev. Lett. 119(1), 011801 (2017). https://doi.org/10.1103/PhysRevLett. 119.011801. arXiv:1704.01122 [hep-ph]

25. B. Lillard, T.M.P. Tait, JHEP 11, 199 (2018). https://doi.org/10. 1007/JHEP11(2018)199 arXiv:1811.03089 [hep-ph]

26. H.S. Lee, W. Yin, Phys. Rev. D 99(1), 015041 (2019). https://doi. org/10.1103/PhysRevD.99.015041. arXiv:1811.04039 [hep-ph] 
27. M. Ardu, L. Di Luzio, G. Landini, A. Strumia, D. Teresi, J.W. Wang, JHEP 11, 090 (2020). https://doi.org/10.1007/ JHEP11(2020)090 arXiv:2007.12663 [hep-ph]

28. W. Yin, JHEP 10, 032 (2020). https://doi.org/10.1007/ JHEP10(2020)032 arXiv:2007.13320 [hep-ph]

29. M. Yamada, T.T. Yanagida, Phys. Lett. B 816, 136267 (2021). $\quad$ https://doi.org/10.1016/j.physletb.2021.136267 arXiv:2101.10350 [hep-ph]

30. J.E. Kim, Phys. Rev. D 31, 1733 (1985). https://doi.org/10.1103/ PhysRevD.31.1733

31. K. Choi, J.E. Kim, Phys. Rev. D 32, 1828 (1985). https://doi.org/ 10.1103/PhysRevD.32.1828

32. K. Harigaya, M. Ibe, K. Schmitz, T.T. Yanagida, Phys. Rev. D 92(7), 075003 (2015). https://doi.org/10.1103/PhysRevD.92. 075003. arXiv:1505.07388 [hep-ph]

33. M. Yamada, T.T. Yanagida, K. Yonekura, Phys. Rev. Lett. 116(5), 051801 (2016). https://doi.org/10.1103/PhysRevLett. 116.051801. arXiv: 1510.06504 [hep-ph]

34. A. Salvio, Phys. Lett. B 808, 135686 (2020). https://doi.org/10. 1016/j.physletb.2020.135686 arXiv:2003.10446 [hep-ph]

35. S. Matsuzaki, K. Yamawaki, JHEP 12, 053 (2015) [erratum: JHEP 11 (2016), 158]. https://doi.org/10.1007/JHEP12(2015)053. arXiv:1508.07688 [hep-ph]

36. P. Draper, D. McKeen, JHEP 04, 127 (2016). https://doi.org/10. 1007/JHEP04(2016)127 arXiv:1602.03604 [hep-ph]

37. P. Agrawal, K. Howe, JHEP 12, 029 (2018). https://doi.org/10. 1007/JHEP12(2018)029 arXiv:1710.04213 [hep-ph]

38. M.K. Gaillard, M.B. Gavela, R. Houtz, P. Quilez, R. Del Rey, Eur. Phys. J. C 78(11), 972 (2018). https://doi.org/10.1140/epjc/ s10052-018-6396-6. arXiv:1805.06465 [hep-ph]

39. T. Gherghetta, M.D. Nguyen, JHEP 12, 094 (2020). https://doi. org/10.1007/JHEP12(2020)094 arXiv:2007.10875 [hep-ph]

40. M. Shaposhnikov, C. Wetterich, Phys. Lett. B 683, 196200 (2010). https://doi.org/10.1016/j.physletb.2009.12.022 arXiv:0912.0208 [hep-th]

41. C. Wetterich, M. Yamada, Phys. Lett. B 770, 268271 (2017). https://doi.org/10.1016/j.physletb.2017.04.049 arXiv: 1612.03069 [hep-th]

42. A. Eichhorn, Y. Hamada, J. Lumma, M. Yamada, Phys. Rev. D 97(8), 086004 (2018). https://doi.org/10.1103/PhysRevD.97. 086004. arXiv:1712.00319 [hep-th]

43. J.M. Pawlowski, M. Reichert, C. Wetterich, M. Yamada, Phys. Rev. D 99(8), 086010 (2019). https://doi.org/10.1103/PhysRevD. 99.086010. arXiv:1811.11706 [hep-th]

44. C. Wetterich, arXiv:1901.04741 [hep-th]

45. K. Johnson, M. Baker, R. Willey, Phys. Rev. 136, B1111-B1119 (1964). https://doi.org/10.1103/PhysRev.136.B1111

46. K. Johnson, R. Willey, M. Baker, Phys. Rev. 163, 1699-1715 (1967). https://doi.org/10.1103/PhysRev.163.1699

47. T. Maskawa, H. Nakajima, Prog. Theor. Phys. 52, 1326-1354 (1974). https://doi.org/10.1143/PTP.52.1326

48. T. Maskawa, H. Nakajima, Prog. Theor. Phys. 54, 860 (1975). https://doi.org/10.1143/PTP.54.860

49. R. Fukuda, T. Kugo, Nucl. Phys. B 117, 250-264 (1976). https:// doi.org/10.1016/0550-3213(76)90572-1

50. V.A. Miransky, Nuovo Cim. A 90, 149-170 (1985). https://doi. org/10.1007/BF02724229

51. K. Yamawaki, M. Bando, K.I. Matumoto, Phys. Rev. Lett. 56, 1335 (1986). https://doi.org/10.1103/PhysRevLett.56.1335

52. M. Bando, K.I. Matumoto, K. Yamawaki, Phys. Lett. B 178, 308312 (1986). https://doi.org/10.1016/0370-2693(86)91516-9

53. K.I. Aoki, M. Bando, K. Hasebe, T. Kugo, H. Nakatani, Prog. Theor. Phys. 82, 1151 (1989). https://doi.org/10.1143/PTP.82. 1151

54. K. Yamawaki, arXiv:hep-ph/9603293 [hep-ph]
55. B. Holdom, Phys. Lett. B 694, 74-79 (2011). https://doi.org/10. 1016/j.physletb.2010.09.037 arXiv:1006.2119 [hep-ph]

56. T. Alanne, S. Blasi, N.A. Dondi, Phys. Rev. Lett. 123(13), 131602 (2019). https://doi.org/10.1103/PhysRevLett.123. 131602. arXiv: 1905.08709 [hep-th]

57. V. Leino, T. Rindlisbacher, K. Rummukainen, F. Sannino, K. Tuominen, Phys. Rev. D 101(7), 074508 (2020). https://doi. org/10.1103/PhysRevD.101.074508. arXiv:1908.04605 [hep-lat]

58. C.N. Leung, S.T. Love, W.A. Bardeen, Nucl. Phys. B 273, 649662 (1986). https://doi.org/10.1016/0550-3213(86)90382-2

59. A.D. Bond, D.F. Litim, Phys. Rev. Lett. 122(21), 211601 (2019). https://doi.org/10.1103/PhysRevLett.122.211601. arXiv:1801.08527 [hep-th]

60. T. Steudtner, JHEP 12, 012 (2020). https://doi.org/10.1007/ JHEP12(2020)012 arXiv:2007.06591 [hep-th]

61. J. Wess, B. Zumino, Phys. Lett. B 37, 95-97 (1971). https://doi. org/10.1016/0370-2693(71)90582-X

62. E. Witten, Nucl. Phys. B 223, 422-432 (1983). https://doi.org/10. 1016/0550-3213(83)90063-9

63. C. Vafa, E. Witten, Nucl. Phys. B 234, 173-188 (1984). https:// doi.org/10.1016/0550-3213(84)90230-X

64. H. Gies, J. Jaeckel, C. Wetterich, Phys. Rev. D 69, 105008 (2004). https://doi.org/10.1103/PhysRevD.69.105008 arXiv:hep-ph/0312034 [hep-ph]

65. M. Shaposhnikov, D. Zenhausern, Phys. Lett. B 671, 162-166 (2009). https://doi.org/10.1016/j.physletb.2008.11.041 arXiv:0809.3406 [hep-th]

66. H. Gies, S. Rechenberger, M.M. Scherer, Eur. Phys. J. C 66, $403-$ 418 (2010). https://doi.org/10.1140/epjc/s10052-010-1257-y arXiv:0907.0327 [hep-th]

67. J. Braun, H. Gies, D.D. Scherer, Phys. Rev. D 83, 085012 (2011). https://doi.org/10.1103/PhysRevD.83.085012 arXiv:1011.1456 [hep-th]

68. F. Bazzocchi, M. Fabbrichesi, R. Percacci, A. Tonero, L. Vecchi, Phys. Lett. B 705, 388-392 (2011). https://doi.org/10.1016/ j.physletb.2011.10.029 arXiv:1105.1968 [hep-ph]

69. C. Wetterich, Phys. Lett. B 718, 573-576 (2012). https://doi.org/ 10.1016/j.physletb.2012.11.020 arXiv:1112.2910 [hep-ph]

70. O. Antipin, M. Gillioz, E. Mølgaard, F. Sannino, Phys. Rev. D 87(12), 125017 (2013). https://doi.org/10.1103/PhysRevD.87. 125017. arXiv:1303.1525 [hep-th]

71. H. Gies, S. Rechenberger, M.M. Scherer, L. Zambelli, Eur. Phys. J. C 73, 2652 (2013). https://doi.org/10.1140/epjc/ s10052-013-2652-y [arXiv:1306.6508 [hep-th]]

72. G. Marques Tavares, M. Schmaltz, W. Skiba, Phys. Rev. D 89(1), 015009 (2014). https://doi.org/10.1103/PhysRevD.89. 015009. arXiv:1308.0025 [hep-ph]

73. S. Abel, A. Mariotti, Phys. Rev. D 89(12), 125018 (2014). https://doi.org/10.1103/PhysRevD.89.125018. arXiv:1312.5335 [hep-ph]

74. A.D. Bond, D.F. Litim, Eur. Phys. J. C 77(6), 429 (2017). [erratum: Eur. Phys. J. C 77 (2017) no.8, 525]. https://doi.org/10.1140/epjc/ s10052-017-4976-5. arXiv:1608.00519 [hep-th]

75. G.M. Pelaggi, A.D. Plascencia, A. Salvio, F. Sannino, J. Smirnov, A. Strumia, Phys. Rev. D 97(9), 095013 (2018). https://doi.org/ 10.1103/PhysRevD.97.095013. arXiv:1708.00437 [hep-ph]

76. A.D. Bond, G. Hiller, K. Kowalska, D.F. Litim, JHEP 08, 004 (2017). https://doi.org/10.1007/JHEP08(2017)004 arXiv:1702.01727 [hep-ph]

77. D. Barducci, M. Fabbrichesi, C.M. Nieto, R. Percacci, V. Skrinjar, JHEP 11, 057 (2018). https://doi.org/10.1007/JHEP11(2018)057 arXiv:1807.05584 [hep-ph]

78. A. Eichhorn, Front. Astron. Space Sci. 5, 47 (2019). https://doi. org/10.3389/fspas.2018.00047 arXiv:1810.07615 [hep-th] 
79. S. Abel, E. Mølgaard, F. Sannino, Phys. Rev. D 99(3), 035030 (2019). https://doi.org/10.1103/PhysRevD.99.035030. arXiv:1812.04856 [hep-ph]

80. H. Pagels, S. Stokar, Phys. Rev. D 20, 2947 (1979). https://doi. org/10.1103/PhysRevD.20.2947

81. V.A. Miransky, V.P. Gusynin, Prog. Theor. Phys. 81, 426-450 (1989). https://doi.org/10.1143/PTP.81.426

82. P.A. Zyla et al. [Particle Data Group], PTEP 2020(8), 083C01 (2020). https://doi.org/10.1093/ptep/ptaa104

83. J. Rantaharju, C. Pica, F. Sannino, Phys. Rev. D 96(1), 014512 (2017). https://doi.org/10.1103/PhysRevD.96.014512. arXiv:1704.03977 [hep-lat]

84. J. Krog, M. Mojaza, F. Sannino, Phys. Rev. D 92(8), 085043 (2015). https://doi.org/10.1103/PhysRevD.92.085043. arXiv:1506.02642 [hep-ph]

85. K.I. Kondo, H. Mino, K. Yamawaki, Phys. Rev. D 39, 2430 (1989). https://doi.org/10.1103/PhysRevD.39.2430

86. T. Appelquist, M. Soldate, T. Takeuchi, L.C.R. Wijewardhana, YCTP-P19-88

87. K.I. Kondo, S. Shuto, K. Yamawaki, Mod. Phys. Lett. A 6, 33853396 (1991). https://doi.org/10.1142/S0217732391003912

88. K.I. Kondo, M. Tanabashi, K. Yamawaki, Prog. Theor. Phys. 89, 1249-1302 (1993). https://doi.org/10.1143/PTP.89.1249. arXiv:hep-ph/9212208 [hep-ph]

89. K.I. Kondo, A. Shibata, M. Tanabashi, K. Yamawaki, Prog. Theor. Phys. 91, 541-572 (1994) [erratum: Prog. Theor. Phys. 93 (1995), 489]. https://doi.org/10.1143/ptp/91.3.541. arXiv:hep-ph/9312322 [hep-ph]

90. K. Kondo, M. Tanabashi, K. Yamawaki, Mod. Phys. Lett. A 8, 2859-2867 (1993). https://doi.org/10.1142/ S021773239300324X

91. M. Harada, Y. Kikukawa, T. Kugo, H. Nakano, Prog. Theor. Phys. 92, 1161-1184 (1994). https://doi.org/10.1143/PTP.92. 1161 arXiv:hep-ph/9407398 [hep-ph]

92. K.I. Kubota, H. Terao, Prog. Theor. Phys. 102, 1163-1179 (1999). https://doi.org/10.1143/PTP.102.1163 arXiv:hep-th/9908062 [hep-th]

93. H. Ishida, S. Matsuzaki, Phys. Lett. B 804, 135390 (2020). https:// doi.org/10.1016/j.physletb.2020.135390 arXiv:1912.09740 [hepph]
94. T. Inagaki, S.D. Odintsov, H. Sakamoto, Astrophys. Space Sci. 360(2), 67 (2015). https://doi.org/10.1007/s10509-015-2584-0. arXiv:1509.03738 [hep-th]

95. T. Inagaki, S.D. Odintsov, H. Sakamoto, Nucl. Phys. B 919, 297-314 (2017). https://doi.org/10.1016/j.nuclphysb.2017. 03.024 arXiv:1611.00210 [hep-ph]

96. T. Inagaki, S.D. Odintsov, H. Sakamoto, EPL 118(2), 29001 (2017). https://doi.org/10.1209/0295-5075/118/29001. arXiv:1706.05489 [hep-th]

97. A.D. Linde, Phys. Rev. D 49, 748-754 (1994). https://doi.org/10. 1103/PhysRevD.49.748 arXiv:astro-ph/9307002 [astro-ph]

98. J.O. Gong, K.S. Jeong, Phys. Rev. D 104(4), 043511 (2021). https://doi.org/10.1103/PhysRevD.104.043511. arXiv:2101.11173 [hep-ph]

99. Y. Hochberg, E. Kuflik, T. Volansky, J.G. Wacker, Phys. Rev. Lett. 113, 171301 (2014). https://doi.org/10.1103/PhysRevLett. 113.171301 arXiv:1402.5143 [hep-ph]

100. Y. Hochberg, E. Kuflik, H. Murayama, T. Volansky, J.G. Wacker, Phys. Rev. Lett. 115(2), 021301 (2015). https://doi.org/10.1103/ PhysRevLett.115.021301. arXiv:1411.3727 [hep-ph]

101. Y. Hochberg, E. Kuflik, H. Murayama, JHEP 05, 090 (2016). https://doi.org/10.1007/JHEP05(2016)090 arXiv:1512.07917 [hep-ph]

102. J. Polchinski, Nucl. Phys. B 231, 269-295 (1984). https://doi.org/ 10.1016/0550-3213(84)90287-6

103. C. Wetterich, Phys. Lett. B 301, 90-94 (1993). https://doi.org/10. 1016/0370-2693(93)90726-X arXiv:1710.05815 [hep-th]

104. T.R. Morris, Int. J. Mod. Phys. A 9, 2411-2450 (1994). https://doi org/10.1142/S0217751X94000972 arXiv:hep-ph/9308265 [hepph]

105. D.F. Litim, Phys. Rev. D 64, 105007 (2001). https://doi.org/10. 1103/PhysRevD.64.105007 arXiv:hep-th/0103195 [hep-th]

106. V. Baluni, Phys. Rev. D 19, 2227-2230 (1979). https://doi.org/ 10.1103/PhysRevD.19.2227

107. J.E. Kim, Phys. Rept. 150, 1-177 (1987). https://doi.org/10.1016/ 0370-1573(87)90017-2 